6. L. Nachbin, A theorem of the Hahn-Banach type for linear transformations, Trans. Amer. Math. Soc. 68 (1950), 28-46.

7. R. S. Phillips, On linear transformations, Trans. Amer. Math. Soc. 48 (1940), 516-541.

8. A. Sobczyk, Projections of the space ( $m)$ on its subspace $\left(c_{0}\right)$. Bull. Amer. Math. Soc. 47 (1941), 938-947.

9. - On the extension of linear transformations, Trans. Amer. Math. Soc. 55 (1944), 153-169.

10. A. E. Taylor, $A$ geometric theorem and its application to biorthogonal systems, Bull. Amer. Math. Soc. 53 (1947), 614-616.

INSTITUTE FOR ADVANCED STUdY

\title{
DENSE ALGEBRAS OF FUNCTIONS IN $L_{p}$
}

\section{R. H. FARRELL 1}

1. Introduction. Several of the known examples of sets of functions dense in $L_{2}(0,1)$ are in fact dense algebras of functions. For example the Walsh functions are closed under pointwise multiplication and therefore the linear combinations of these functions form an algebra. The linear space generated by $1,\{\sin (n \theta), n \geqq 1\},\{\cos (n \theta), n \geqq 1\}$ is again an algebra. It is suggested then that perhaps the conditions of the Stone-Weierstrass theorem can be given a measure-theoretic interpretation which would allow proof of a corresponding theorem in the $L_{p}$ spaces. It is the purpose of this paper to state and prove such a theorem. We will assume that a locally-compact topological space $X$ is given and $B$ is the class of Baire sets of $X$. We assume $B$ is a $\sigma$-algebra containing $X$; therefore that $X$ is a Baire set.

We prove the following theorem.

THEOREM. Let $\mu$ be a real valued $\sigma$-finite Baire measure on $X, \bigotimes$. Let $E$ be an algebra of real valued essentially bounded Baire measurable functions. Suppose $1 \leqq p<\infty$ is given and $E \subset L_{p}(\mu)$. Suppose

(1) there is an $h \in E$ such that $h>0$ a.e. $\mu$;

(2) there is given a base $\left\{U_{\alpha}\right\}$ for the open Baire sets of $X$ such that if $U_{\alpha} \cap U_{\beta}=\Phi$ then for some $h \in E, h \leqq 0$ a.e. $\mu$ on $U_{\alpha}$ and $h>0$ a.e. $\mu$ on $U_{\beta}$. Then $E$ is dense in $L_{p}(\mu)$.

The proof is given in the next section. In the final section of this paper additional examples are given.

Received by the editors February 25, 1961.

1 Research sponsored by the Office of Naval Research under contract No. Nonr401(03). 
For a discussion of locally compact spaces, Baire measures and regularity of measures, see Halmos [1].

2. Proof of the theorem. We show that if $I$ is a continuous linear functional on $L_{p}(\mu)$ such that $I(h)=0$ for every $h \in E$ then $I$ is identically zero. This implies the closure $\bar{E}$ of $E$ in $L_{p}(\mu)$ is $L_{p}(\mu)$ by virture of the Hahn-Banach theorem.

If $I$ is a continuous linear functional on $L_{p}(\mu), 1<p<\infty$, then there is a function $f \in L_{q}(\mu)$ such that $I(g)=\int f g d \mu$ for every $g \in L_{p}(\mu)$. As $\mu$ is assumed $\sigma$-finite, in case $p=1$, such a function $f \in L_{\infty}(\mu)$ may be found. Let $A_{+}=\{x \mid f(x)>0\}$ and $A_{-}=\{x \mid f(x)<0\}$. By hypothesis there is a function $h_{0} \in E, h_{0}>0$ a.e. $\mu$. Suppose $I(g)=0$ for every $g \in E$ but $I \neq 0$. Then $\mu\left(A_{+}\right)>0$ and $\mu\left(A_{-}\right)>0$. For if $\mu\left(A_{+}\right)=0$ then $I\left(h_{0}\right)=\int_{A_{-}}\left|f h_{0}\right| d \mu=0$ which implies $\mu\left(A_{-}\right)=0$. Similarly if $\mu\left(A_{-}\right)=0$ then $\mu\left(A_{+}\right)=0$. We now show the hypothesis $I(g)=0$ for every $g \in E$ but $\mu\left(A_{+}\right)>0, \mu\left(A_{-}\right)>0$ leads to a contradiction. We do this by showing there must be $h \in \bar{E}$, the $L_{p}$ closure of $E$, such that $h>0$ a.e. on $A_{+}, h<0$ a.e. on $A_{-}$. The first part of the argument reduces the $\sigma$-finite case to the case $\mu$ is a finite measure. Then by arguments similar to those used to develop the theory of the Daniell integral the function $h$ is constructed. See Loomis [2] for a discussion of the Daniell integral.

To reduce the proof to the case $\mu$ is a finite measure, let $h_{0} \in E$, $h_{0}>0$ a.e. $\mu$. Define a measure $\nu$ by $\nu(A)=\int_{A} h_{0}^{p} d \mu$. Then $\nu$ is a finite measure. Since $h_{0}$ is essentially bounded $L_{p}(\mu) \subset L_{p}(\nu)$. Therefore $E \subset L_{p}(\nu)$. Supposing the theorem proven for finite measures there is an $h \in \bar{E}$, the $L_{p}(\nu)$ closure of $E$, such that $h>0$ a.e. $\nu$ on $A_{+}$and $h<0$ a.e. $\nu$ on $A_{-}$. Since $h_{0}>0$ a.e. $\mu, h>0$ a.e. $\mu$ on $A_{+}$and $h<0$ a.e. $\mu$ on $A_{-}$follows. Next, there is a sequence $\left\{h_{n}, n \geqq 1\right\} \subset E$ such that $\lim _{n \rightarrow \infty} \int\left|h_{n}-h\right|^{p} h_{0}^{p} d \mu=0$. Therefore $h_{n} h_{0} \rightarrow h h_{0}$ in $L_{p}(\mu)$. But $h h_{0}>0$ a.e. $\mu$ on $A_{+}$and $h h_{0}<0$ a.e. $\mu$ on $A_{-}$. Therefore $h h_{0} \in \bar{E}$ is the required function.

We suppose in the sequel that $\mu$ is a finite measure, i.e., $\mu(X)<\infty$. Suppose $\bar{E}$ is the $L_{p}(\mu)$ closure of $E$. Since $\mu$ is a finite measure $\bar{E}$ is closed under uniform pointwise limits. Therefore if $h \in E,|h| \in \bar{E}$, for $h$ is essentially bounded. Then it follows for every $h \in \bar{E},|h| \in \bar{E}$. For if $\left\{h_{n}, n \geqq 1\right\} \subset E, h_{n} \rightarrow h$ in $L_{p}(\mu)$, then as $\| h_{n}|-| h|| \leqq\left|h_{n}-h\right|$, $\left|h_{n}\right| \rightarrow|h|$ in $L_{p}(\mu)$. But $\left|h_{n}\right| \in \bar{E}$ for $n \geqq 1$. Therefore $\bar{E}$ is closed under max and min.

To prove the theorem it is sufficient to show that for every pair $A$ and $B$ of disjoint Baire sets there is a function $k \in \vec{E}$ such that $k=0$ a.e. $\mu$ on $B$ and $k=h_{0}>0$ a.e. on $A$. To do this we use the regu- 
larity of Baire measures and the hypothesis that $\left\{U_{\alpha}\right\}$ is a base for the open sets of $X$.

Suppose then $C_{1}$ and $C_{2}$ are disjoint compact sets. Then in the base there are open sets $U_{1}, \cdots, U_{m}$ and $V_{1}, \cdots, V_{n}$ such that $U_{i} \cap V_{j}=\Phi, i=1, \cdots, m, j=1, \cdots, n$ and $C_{1} \subset \bigcup_{i=1}^{m} U_{i}, C_{2} \subset \bigcup_{j=1}^{n} V_{j}$. By hypothesis there is a family $h_{i j}$ of functions in $E$ such that $h_{i j}>0$ a.e. on $U_{i}, h_{i j} \leqq 0$ a.e. on $V_{j}$. Let $h=\min _{j} \max _{i} h_{i j}$. Then $h \in \bar{E}, h>0$ a.e. on $C_{1}$ and $h \leqq 0$ a.e. on $C_{2}$. Since $h^{+} \in \bar{E}$, we may obtain $h^{*} \in \bar{E}$, $h^{*}=\min \left(h_{0}, h^{+}\right)$such that $h^{*}>0$ a.e. on $C_{1}, h^{*}=0$ a.e. on $C_{2}$, and $0 \leqq h^{*} \leqq h_{0}$ a.e.

Suppose $A$ has positive measure, $C$ is compact, and $A \cap C=\Phi$. Then since $\mu$ is finite there exists a sequence $\left\{C_{n}\right\}$ of compact sets such that $\bigcup_{n=1}^{\infty} C_{n} \subset A$ and $\mu(A)=\mu\left(\cup_{n=1}^{\infty} C_{n}\right)$. Let $h_{n}$ be in $\bar{E}, 0 \leqq h_{n}$ $\leqq h_{0}$ a.e., $h_{n}>0$ a.e. on $C_{n}$, and $h_{n}=0$ a.e. on $C$. Then $k=\sup _{n} h_{n} \in \bar{E}$, $k>0$ a.e. on $A, k=0$ a.e. on $C$, and $0 \leqq k \leqq h_{0}$ a.e.

Next, $\sup _{n} \min \left(h_{0}, n k\right) \in \bar{E}$. We may thus suppose $k=h_{0}$ a.e. on $A$ and that $k=0$ a.e. on $C$. Now let $A, B$ be disjoint sets of positive measure. Let $\left\{C_{n}, n \geqq 1\right\}$ be a sequence of compact sets, $\cup_{n=1}^{\infty} C_{n} \subset B$ and $\mu(B)=\mu\left(\cup_{n=1}^{\infty} C_{n}\right)$. Then for each $n$ there is a $k_{n}$ such that

$$
\begin{aligned}
k_{n} & =h_{0} \text { a.e. on } A, \\
k_{n} & =0 \text { a.e. on } C_{n}, \\
0 & \leqq k_{n} \leqq h_{0} \text { a.e. }
\end{aligned}
$$

Then $k^{*}=\inf _{n} k_{n}$ is the required function. The proof is complete.

3. Examples. It should be noted that if $E \subset L_{p}(\mu)$ then for every $p \leqq r \leqq \infty, E \subset L_{r}(\mu)$. If $\mu$ is a finite measure then for every $1 \leqq r \leqq p$, $E \subset L_{r}(\mu)$.

Corollary 1. Suppose $E$ is the algebra of real valued continuous functions with compact support. If $X$ is locally compact and $X$ is a Baire set then for every Baire measure $\mu, E$ is dense in $L_{p}(\mu), 1 \leqq p<\infty$.

Proof. The open Baire sets with compact closure form a base for the topology. Since $E \subset L_{p}(\mu)$ for every $1 \leqq p<\infty$, hypothesis (2) is satisfied. Let $\bar{E}$ be the $L_{p}(\mu)$ closure of $E$. Let $C_{1} \subset C_{2} \subset \cdots$ be an increasing sequence of compact Baire sets of positive measure, $X=\cup_{n=1}^{\infty} C_{n}$. Let $f_{n}$ be a continuous function such that $0 \leqq f_{n} \leqq 1$, $f_{n}(x)=1$ if $x \in C_{n}, f_{n}(x)=0$ if $x \notin C_{n+1}$. Then $f_{n} \in E$ for $n \geqq 1$. Let $a_{n}=\mu\left(C_{n}\right)$. Since $\mu$ is a Baire measure, $a_{n}<\infty$. $f=\sum_{n-1}^{\infty} f_{n} / n^{2} a_{n+1} \in \bar{E}$, $f>0$ a.e., and $f$ is bounded. The algebra generated by $E$ and $f$ is contained in $\bar{E}$. By the theorem this algebra is dense in $L_{p}(\mu)$. The proof is complete. 
Corollary 2. Suppose $X$ is a locally compact space, $X$ a Baire set, and $E$ an algebra of continuous real valued functions vanishing at infinity. Let $\mu$ be a Baire measure on $X$. Suppose there is an $f \in E$ such that $f>0$ a.e. $\mu$. If $E \subset L_{p}(\mu)$ and $E$ is uniformly dense in the algebra of all continuous functions vanishing at infinity then $E$ is dense in $L_{p}(\mu)$.

To prove this corollary let $\nu$ be defined by $\nu(A)=\int_{A} f^{p} d \mu$. Then $E \subset L_{p}(\nu)$ and by Corollary $1, \bar{E}=L_{p}(\nu)$. For the uniform closure of $E$ is in $L_{p}(\nu)$ and this contains all functions vanishing at infinity.

Suppose $E$ not dense in $L_{p}(\mu)$. Repeating the first part of the proof of the theorem a contradiction is obtained.

If the space $X$ is a complete separable metric space the theorem remains true if $B$ is the least $\sigma$-algebra containing the open sets. To see this we observe that the assumption of local compactness was used only to allow approximation of measurable sets by compact sets. This kind of approximation may also be used in case $X$ is a complete separable metric space as is stated in the following lemma. See Prohorov [3].

Lemma. Let $\mu$ be a finite measure on the Borel sets $B$ of the complete separable metric space $X$. Given $\epsilon>0$ and $A \in B$ there is a compact set $C \in B$ such that $C \subset A$ and $\mu(A-C)<\epsilon$.

In certain problems we may wish to allow measurable complex valued functions. A possible modification of the hypotheses of the theorem is as follows: (3) The algebra $E$ is closed under conjugation, i.e., if $f \in E$ then $\bar{f} \in E$. Let $R E$ be the functions which are real parts of functions in $E$ and $I E$ be the functions which are imaginary parts of functions in $E$. The hypothesis (3) implies $R E$ is an algebra. Always $R E=I E$. Suppose hypotheses (1) and (2) of the theorem are satisfied by $R E$ as well as (3) satisfied by $E$. Then $E$ is dense.

As another example we state without proof

CoRollary 3. Suppose $F$ is a strictly increasing and bounded function on $[0,1]$. Let $\mu$ be a finite measure defined on the Borel subsets of $[0,1]$. Then for every $p, 1 \leqq p<\infty$ the algebra generated by 1 and $F$ is dense in $L_{p}(\mu)$.

In applications to statistics the concept of bounded completeness has become important. A family $\mathcal{F}$ of probability measures on a space $X, B$ is given. If for every bounded measurable function $f, 0=\int f d \mu$ for every $\mu \in \mathcal{F}$ implies $f=0$ a.e. for every $\mu \in \mathcal{F}$ then the family $\mathcal{F}$ is said to be boundedly complete. There is of ten a $\sigma$-finite measure $\nu$ such that each $\mu \in \mathcal{F}$ is absolutely continuous with respect to $\nu$. If $\mathcal{F}^{*}$ is the 
class of Radon-Nikodym derivatives of the measures in $F$ relative to $\nu$ then the hypothesis of bounded completeness of $\mathcal{F}$ is equivalent to $\mathcal{F}^{*}$ being dense in $L_{1}(\nu)$. In many of the common examples that arise in statistics the linear space generated by $\mathcal{F}^{*}$ is an algebra satisfying the hypotheses of the theorem. If this is true we obtain an even stronger result.

CoRollary 4. Suppose $X$ locally compact and $\mu$ a totally $\sigma$-finite Baire measure. Let $E$ be an algebra of essentially bounded Baire measurable functions, $E \subset L_{1}(\mu)$. Suppose $E$ satisfies (1) and (2) of the theorem. Suppose $f$ is Baire measurable, $\int|f g| d \mu<\infty$ for every $g \in E$ and $\int f g d \mu=0$ for every $g \in E$. Then $f=0$ a.e. $\mu$.

The proof is exactly as for the theorem. Take $h_{0} \in E, h_{0}>0$ a.e. $\mu$ and work relative to the finite measure $\nu, \nu(A)=\int_{\Lambda} h_{0}(x) d \mu(x)$. Let $E^{*}$ be the uniform closure of $E$. Then $E^{*}$ is in $L_{\infty}(\nu), f \in L_{1}(\nu)$ and the remainder of the proof goes through as before.

\section{BIBLIOGRAPHY}

1. Paul R. Halmos, Measure theory, D. Van Nostrand Company, New York, 1950.

2. Lynn H. Loomis, An introduction to abstract harmonic analysis, D. Van Nostrand Company, New York, 1953.

3. Yu. V. Prohorov, Convergence of random processes and limit theorems in probability, Theor. Probability Appl. 1 (1956), 157-214.

CoRNell University 